\title{
Fault Localisation for WS-BPEL Programs based on Predicate Switching and Program Slicing
}

\author{
Chang-ai Sun ${ }^{\mathrm{a}, *}$, Yufeng Ran ${ }^{\mathrm{a}}$, Caiyun Zheng ${ }^{\mathrm{a}}$, Huai Liu ${ }^{\mathrm{b}}$, Dave Towey ${ }^{\mathrm{c}}$, Xiangyu Zhang ${ }^{\mathrm{d}}$ \\ ${ }^{a}$ School of Computer and Communication Engineering, University of Science and Technology Beijing, Beijing 100083, China \\ ${ }^{b}$ College of Engineering and Science, Victoria University, Melbourne 8001 VIC, Australia \\ ${ }^{c}$ School of Computer Science, University of Nottingham Ningbo China, Zhejiang, 315100, China \\ ${ }^{d}$ Department of Computer Science, Purdue University, West Lafayette, Indiana, USA
}

\begin{abstract}
Service-Oriented Architecture (SOA) enables the coordination of multiple loosely coupled services. This allows users to choose any service provided by the SOA without knowing implementation details, thus making coding easier and more flexible. Web services are basic units of SOA. However, the functionality of a single Web service is limited, and usually cannot completely satisfy the actual demand. Hence, it is necessary to coordinate multiple independent Web services to achieve complex business processes. Business Process Execution Language for Web Services (WS-BPEL) makes the coordination possible, by helping the integration of multiple Web services and providing an interface for users to invoke. When coordinating these services, however, illegal or faulty operations may be encountered, but current tools are not yet powerful enough to support the localisation and removal of these problems. In this paper, we propose a fault localisation technique for WS-BPEL programs based on predicate switching and program slicing, allowing developers to more precisely locate the suspicious faulty code. Case studies were conducted to investigate the effectiveness of the proposed technique, which was compared with predicate switching only, slicing only, and one existing fault localisation technique, namely Tarantula. The experimental results show that the proposed technique has a higher fault localisation effectiveness and precision than the baseline techniques.
\end{abstract}

Keywords: Fault localisation, Debugging, Business Process Execution Language for Web Services, Web Services

\section{Introduction}

In recent years, Service-Oriented Architecture (SOA) [1] has been widely adopted to develop distributed applications in various domains. Web services, as basic units in SOA, are often developed and owned by a third party, and are published and deployed in an open and dynamic environment. Since a single Web service normally provides limited functionalities, multiple Web services are coordinated to implement complex and flexible business processes. Business Process Execution Language for Web Services (WS-BPEL) [2] is a widely recognised language for service compositions. In the context of WS-BPEL, all communications among Web services are via standard eXtensible Markup Language (XML) messages [3]. Compared with traditional programs written in C or Java, WS-BPEL programs

\footnotetext{
${ }^{*}$ Corresponding author

Email address: casun@ustb.edu.cn (Chang-ai Sun)
}

have many new features. For instance, a WS-BPEL program is represented as an XML file, and dynamic behaviours of the program are embedded into structural XML elements; Web services composed by WS-BPEL programs may be implemented in hybrid programming languages; and WS-BPEL supports concurrency and synchronisation that is not common in the traditional programs.

The above unique features make the debugging of WS-BPEL programs significantly different from that of traditional programs. Unfortunately, very little research in this direction has been reported. In our previous work [4], we presented a block-based fault localisation framework for WS-BPEL programs, and synthesised the three well-known spectrum-based fault localisation techniques: Tarantula [5], Set-Union [6], and Code Coverage $[7,8]$. We also conducted an empirical study to evaluate the effectiveness of the synthesized WS-BPEL-specific fault localisation techniques. The result showed that the Tarantula technique was the 
most effective technique, demonstrated by the highest 86 accuracy in localising the blocks that contain the faulty 87 statement. However, these techniques could only report 88 those suspicious faulty blocks without a deeper analysis 89 inside the suspicious block.

There also exist some development platforms for 91 WS-BPEL, such as ActiveBPEL Designer [9] and 92 Eclipse BPEL Designer [10]. Unfortunately, these plat- 93 forms usually only provide support for WS-BPEL syn- 94 tax checking, while the assistance with logical errors 95 that most developers expect is missing. If such assis- 96 tance were available, perhaps as a plug-in to the plat- 97 form, the debugging efficiency might be substantially 98 improved.

In this work, we attempt to develop a technique 100 to further improve the effectiveness and efficiency of 101 fault localisation for WS-BPEL programs. In particu- 102 lar, we propose a new fault localisation technique for 103 WS-BPEL programs, based on two popularly used tech- 104 niques, namely predicate switching [11] and program 105 slicing [12]. The proposed technique first employs pred- 106 icate switching to narrow the range of blocks to be 107 checked for the fault localisation, and then makes use 108 of slicing to go more deeply into the block for a more 109 precise analysis of the fault. In particular, we focus 110 on the following unique challenges in applying predi- 111 cate switching and program slicing into WS-BPEL pro- 112 grams.

- Predicate switching for WS-BPEL programs: For ${ }_{114}$ $\mathrm{C}, \mathrm{C}++$, or Java programs, predicate switching ${ }_{115}$ is normally implemented through instrumentation. ${ }_{116}$ However, WS-BPEL programs are basically the ${ }_{117}$ workflow specifications based on XML, and their ${ }_{118}$ executions normally rely on a specific interpreter. For instance, Apache ODE [13] is a popular WSBPEL engine that compiles all standard BPEL elements: the compiled VxBPEL is represented as an object model containing all necessary resources for execution. A runtime component is responsible for the execution of compiled processes. Such an execution mode means that dynamic changes to WS-BPEL programs are not allowed. In contrast, the original implementation of predicate switching for $\mathrm{C}$ programs is based on Valgrind ${ }^{1}$. Valgrind supports dynamic instrumentation by calling the instrumentation functions. These functions, in turn, instrument the provided basic block and return the new basic block to the Valgrind kernel (refer to [11] for more details). Clearly, because no

\footnotetext{
${ }^{1}$ Valgrind is a well-known memory debugger and profiler for x 86- ${ }^{135}$ kubyx binaries. For more details, please refer to: http://valgrind.org/ 136
}

interfaces are reserved for calling instrumentation functions, it is not possible to implement dynamic instrumentation in Apache ODE. Furthermore, in this context, instrumentation is not a suitable solution for WS-BPEL programs. In order to implement an instrumentation function in WS-BPEL programs, it would be necessary to make significant modifications to the original WS-BPEL programs. Such modifications would definitely affect the whole program, including the partner link, variable, and interaction sections. Furthermore, these modifications would change the semantics of the original program, which violates the fundamental principle of instrumentation technique. On the contrary, the original instrumentation for predicate switching in C programs (again refer to [11]) does not face this challenge, because it implements the instrumentation functions in binaries, and the modifications to the original program include only introducing a new basic block. Finally, collecting the execution traces of instrumentation for WSBPEL programs is also challenging. It is easy to collect execution traces in the context of $\mathrm{C}$ programs, which can be done by writing data into a file or memory. In contrast, WS-BPEL programs normally return a response message through a specific activity (i.e. reply), and there is no channel for throwing trace data.

- Dynamic program slicing for WS-BPEL programs: Variables in WS-BPEL programs can be either an atomic data type or a complex composite type whose definitions are normally distributed in various namespaces represented in XML files. Thus, dynamic slicing of WS-BPEL programs must be able to deal with recursive parsing and querying of composite variables. Furthermore, it is necessary to analyse the interpreter's logs in order to obtain the execution traces of the WS-BPEL program. These issues all pose challenges for the dynamic slicing of WS-BPEL programs.

Based on some new concepts, the above challenges are effectively addressed in our proposed technique.

In order to evaluate the performance of the proposed technique, we conducted a comprehensive empirical study where three WS-BPEL programs were used as object programs, and a total of 166 mutated versions were used to simulate various faults. The effectiveness and precision of the new technique were compared with the Tarantula technique, which had shown the best performance in fault localisation for WS-BPEL programs in our previous work [4]. Experimental results showed 
that the proposed technique demonstrates better effectiveness and higher precision than the previous technique.

The rest of this paper is organised as follows. Section 2 introduces underlying concepts of WS-BPEL, and some related fault localisation techniques. Section 3 describes the main idea of our new fault localisation technique. Details on how to apply the technique to WS-BPEL programs are also discussed. Section 4 describes an empirical study that was conducted to evaluate the proposed fault localisation technique. Section 5 presents the results of the empirical study, and offers an analysis. Section 6 discusses some important work related to our study. Finally, Section 7 concludes the paper, and discusses the future work.

\section{Background}

\subsection{WS-BPEL}

The Business Process Execution Language for Web ${ }^{189}$ Service (WS-BPEL) is a widely used language for com- ${ }^{190}$ posing Web services [2]. It can integrate multiple Web ${ }^{191}$ services to form a business process, and make this avail- 192 able in the form of Web services [4]. In this sense, a 193 WS-BPEL Web service is actually a composite Web ser- ${ }^{194}$ vice whose invocation interface can be described using 195 the Web Service Description Language (WSDL) [14]. ${ }^{196}$ WS-BPEL programs aim to integrate all Web services ${ }^{197}$ in one line to reduce the program redundancy, without 198 requiring details of the actual implementation of the ser- 199 vice [15].

WS-BPEL programs are usually composed of four sections: variable section, partner link section, handler section, and interaction section [4]. The variable section defines input and output messages. The partner link section describes the relationship among the WS-BPEL process and invoked Web services. The handler section declares the handlers when an exception or specific event occurs. The interaction section describes how external Web Services are coordinated to execute a business process.

The basic interaction unit of a WS-BPEL program is an activity, which can be either a basic activity or a 202 structured activity. Basic activities describe an atomic 203 execution step (such as assign, invoke, receive, reply, 204 throw, wait, and empty); and structured activities are 205 composed of several basic activities or other structured 206 activities (such as sequence, switch, while, flow, and 207 pick). Figure 1 shows an interaction segment of a WS- 208 BPEL program.

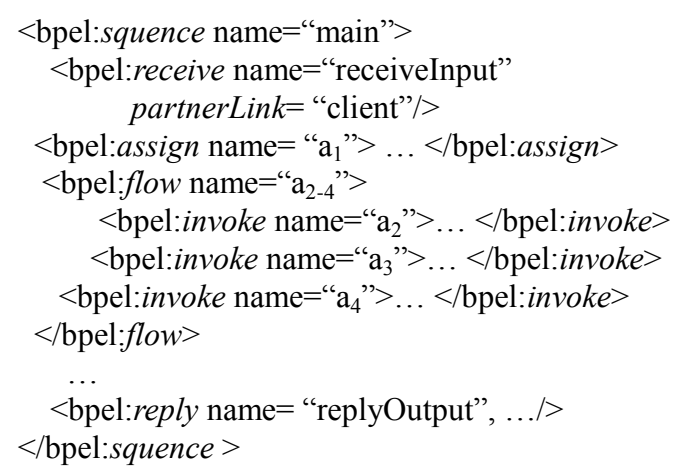

Figure 1: Illustration of the interaction of a WS-BPEL Program

\section{5} program analysis, on program execution, on predicates, and also using data mining or machine learning [17]. Among them, spectrum-based fault localisation is a family of fault localisation techniques based on program execution that counts the executions of program elements in different executions, and uses the ratio of a program element being exercised in a failed execution and the one in a passed execution to calculate the suspiciousness of the program element. We next introduce one representative spectrum-based technique that will be included for evaluation in our experiments reported in Section 4.

Jones [5] proposed a program execution-based fault localisation technique, using statistics, called Tarantula. Tarantula involves multiple test cases and executions, recording the pass and fail status for each program element a test case executes. The suspiciousness value is calculated according to Formula 1 below:

$$
\operatorname{suspicion}(s)=\frac{\frac{\text { failed }(s)}{\text { totalfailed }}}{\frac{\text { passed }(s)}{\text { totalpassed }}+\frac{\text { failed }(s)}{\text { totalfailed }}},
$$

where $\operatorname{passed}(s)$ is the number of test cases that have executed the program element $s$ with the output as expected; failed $(s)$ is the number of test cases that have executed the program element $s$ with the output not as expected; totalpassed is the total number of passing test cases; and totalfailed is the total number of failing test cases. Program elements are ranked according to these suspicion values, with higher values indicating a higher 
likelihood of containing the fault. Limitations of the 260 Tarantula method include that it requires a large set of 261 tests, with the pass or fail status known, and that if ei- 262 ther totalpassed or totalfailed is zero, then the formula 263 is invalid.

In our previous work [4], we evaluated the perfor- 265 mance of three traditional spectrum-based fault local- 266 isation techniques, namely Tarantula, Set-Union, and Code Coverage, on two WS-BPEL programs. The fault ${ }_{267}$ localisation effectiveness was mainly measured by the correctness percentage, which refers to the percentage of possible position sets that really contain the faulty statements. For one program (SupplyChain), Tarantula, Set-Union, and Code Coverage can successfully locate $7(53.8 \%), 7(53.8 \%)$, and $5(38.5 \%)$ of 13 faults, respectively. For the other program (SmartShelf), Tarantula, Set-Union, and Code Coverage can successfully locate $10(50 \%), 8(40 \%)$, and $8(40 \%)$ of 20 faults, respectively. Such observations implied that Tarantula was the most effective among these three fault localisation techniques. However, it should be noted that the performance of Tarantula (as well as Set-Union and Code Coverage) on WS-BPEL programs is not as good as that on traditional programs. For instance, Tarantula can achieve a score of $90 \%$ (i.e. the fault was found by examining less than $10 \%$ of the executed code) for the $55.7 \%$ faulty versions in seven $\mathrm{C}$ programs in the Siements suite [18]. In other words, there is a need for more advanced techniques specifically for localising faults in WS-BPEL programs.

In order to further improve the fault localisation ef fectiveness and efficiency of WS-BPEL programs, we explore predicate switching and program slicing-based fault localisation for WS-BPEL programs, and address the key issues of the proposed technique. We also compare the fault localisation effectiveness and efficiency of the proposed technique with that of predicate switching only, slicing only, and Tarantula, since Tarantula was evaluated to be the most effective technique in our previous work [4].

\section{BPELswice: A Fault Localisation Technique for 300 WS-BPEL Programs}

Normally, traditional programs written in Java or C ${ }^{302}$ focus more on trivial operations on various data structures, from the simple data types such as char, integer, 304 boolean, and real to the complex data types such as ar- 305 ray, struct/union, pointer, and their composites. Differ- 306 ent from them, WS-BPEL programs specify a work- 307 flow with coarse-grained activities, which usually in- 308 volve simple operations such as invoking an external 309
Web services or a variable assignment. The transitions between the activities are implemented through some common control logic such as sequence, optional, loop, and also newly introduced concurrency and synchronisation. These unique features of WS-BPEL programs pose challenges for fault localisation, and thus call for new techniques.

\subsection{Overview}

A fault is considered to be detected when a test case causes the program to have an incorrect output. The fault-revealing test case is also called the failed test case, the counterpart of which is called the successful test case that results in correct output. Each failed test case corresponds to the execution of a particular path, which can help us precisely localise the fault.

A typical program usually contains a number of branches, as part of its logical structure. These branches are controlled by some conditional slice, called a predicate, which evaluates to either true or false. If we force a predicate to change its true or false status, then we have a process called predicate switching. The goal of this process is to find a predicate that has strong influence on the data flow, and if the branch outcome of the predicate is switched and execution is continued, the output of the program may be changed from "incorrect" to "as expected", thereby providing a valuable clue as to the location of the fault. Such a predicate, if it exists, is called a critical predicate. Statements that change the values of variables related to this critical predicate, which exist between it and the start of the program, are called the backward slice. Because the critical predicate may be strongly influenced by the backward slice, the analysis is necessary, and may provide further guidance to precisely locate the actual fault which caused the predicate's incorrect status.

We hereby propose a fault localisation technique for WS-BPEL programs based on the predicate switching and backward slices, which is abbreviated as BPELswice in the rest of the paper. Figure 2 shows the basic framework of the technique, for which it is assumed that at least one test case demonstrates the presence of a fault in the WS-BPEL program.

As shown in Figure 2, BPELswice includes the following five major steps:

1. Parsing the WS-BPEL program enables an enumeration of all the possible paths through the program and all predicates associated with each path, which facilitates the backward slice analysis.

2. Predicate switching revises a predicate of the WSBPEL program and then deploys the revised WS- 


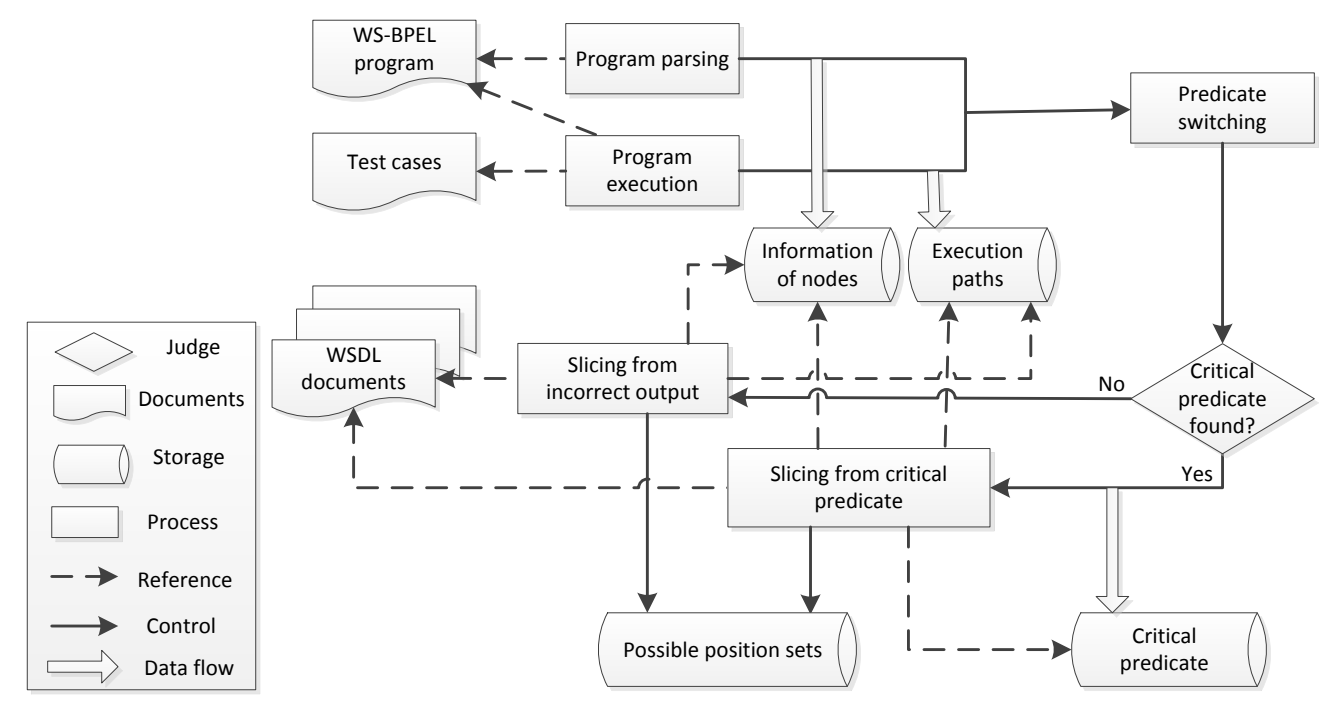

Figure 2: Framework of the proposed BPELswice technique

BPEL program for execution. In order to exe- 338 cute the different conditional part, this stage ac- ${ }_{339}$ tually changes the predicate value from "true" to ${ }_{340}$ "false", or vice versa. The switching is imple- ${ }_{341}$ mented by negating the predicate of the original ${ }_{342}$ WS-BPEL program, and accordingly a WS-BPEL ${ }_{343}$ program variant is derived in this stage. During the ${ }_{344}$ switching, we can use some strategies to decide the ${ }_{345}$ switching ordering of the predicates.

3. Execution includes passing the failed test cases as 347 input for the deployed WS-BPEL program variant 348 and obtaining the actual output. This stage usu- 349 ally involves a WS-BPEL engine, such as Apache ODE [13], which produces a series of events in a ${ }^{350}$ $\log$ file. Through the analysis of the log file, one can extract all executed path nodes and variable's ${ }^{351}$ values during the current execution.

4. Evaluation involves comparison of the actual output with what was expected (i.e. the oracle). In this ${ }_{354}$ stage, the main goal is to observe how the predicate 355 switching impacts on the output of the revised WS- 356 BPEL program. If the output is different from the ${ }_{357}$ expected output, then we continue to switch the re- 358 maining ordered predicates (i.e. repeat predicate 359 switching and evaluation steps). This switching 360 process is repeated until the actual output becomes 361 the same as the oracle, at which moment the crit- 362 ical predicate is found and then we can go to the ${ }_{363}$ next, slicing, step.

5. Slicing aims to further reduce the possible position set of the fault. In this step, the main task is to find backward slices between the critical predicate and the start node (i.e. the receiveInput node of the WS-BPEL program). Note that the backward slices are those nearest statements that directly affect the values of the elementary variables in the critical predicate. Through comparing the values achieved at run-time with what we expected, we can find the variable and its statement node that are different from the expected ones.

Each major step is detailed in the following sections.

\subsection{Parsing the WS-BPEL program}

It is necessary to parse the WS-BPEL program for user's understanding of its structure. The document root is critical for the parsing, and is the entry (the so-called "main node") to the program. Typically, there are two traverse methods to read a program: Depth-First Traversal and Breadth-First Traversal. The choice of traversal method does not affect the information we obtain from a WS-BPEL program. Once all the WS-BPEL node information has been obtained, it is inserted on a JTree [19], which shows all paths through the program.

The computation in a WS-BPEL program which calculates an output can be divided into two categories: the 
Data Part (DP) and the Select Part (SP) [11]. The Data 399 Part includes execution instructions which help in com- 400 puting data values or defining variables that are involved 401 in computing the output of the program. Sometimes 402 these are important parts for backward slices. The Se- 403 lect Part includes instructions which cause the selection 404 of program branches - for example, in the conditionals 405 of "if", "switch", and "while" activities. Different pro- 406 gram executions may involve different data slices, lead- ${ }_{407}$ ing to generation of differently computed output values. 408 Sometimes the conditions in Select Parts may depend 409 on the values computed in the Data Parts. Furthermore, 410 because the output values are determined by the selec- ${ }_{411}$ tion of program branches, it is necessary to analyse the ${ }_{412}$ Select Parts to locate the faulty code. Figure 3 illustrates 413 the Data Part and the Select Part in a sample WS-BPEL 414 program.

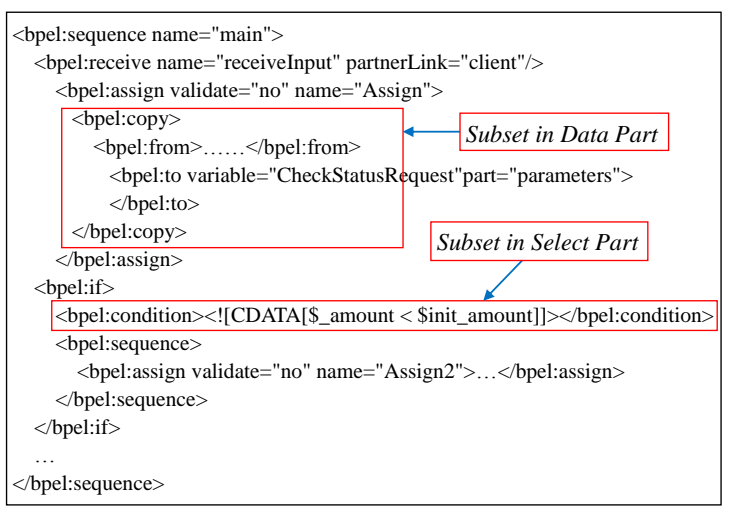

Figure 3: Data Part and Select Part in WS-BPEL Program

In summary, the output of a program is influenced by ${ }_{431}$ two things in the code: the data dependence part, and ${ }_{432}$ the selection part. Altering code in the selection part ${ }_{433}$ may lead to a change in the output, which may enable ${ }_{434}$ us to track down where in the code an error was made. ${ }_{435}$ The selection part of the code which controls the flow ${ }_{436}$ is called a predicate, and a predicate whose outcome is changed, e.g., from false to true, resulting in a change ${ }^{437}$ to the overall program output changing to the expected output, is called a critical predicate. The question of ${ }^{439}$ how to find the critical predicate will be addressed in 440 the following.

\subsection{Predicate Switching}

We aim to reduce the possible location range of the fault using the critical predicate technique [11], a central part of which is predicate switching. Predicate switch- 445 ing involves going through a sequence of predicates in 446 any executed path, switching the boolean status of each ${ }_{447}$ (e.g. changing "true" to "false"), and examining the impact on the output: if the output changes to the expected one, then the critical predicate is the predicate whose status was most recently switched.

Before starting to switch predicates, we first order them, to reduce the amount of time required to identify the critical predicate. We hereby illustrate one typical and widely-used ordering strategy, called Last Executed First Switching ordering (LEFS) [11].

The LEFS ordering strategy is based on the observation that a failure (that is, the incorrect output different from expectation) usually occurs not far away from the execution of the faulty code. This leads to the decision to reverse the order of predicates such that the first one to be checked will be the most recently executed. Suppose that a test case $t$ caused a failure of a WS-BPEL program. We first identify the sequence $\sigma_{n}$ of predicates when executing $t$ on the program, saying $p_{1}, p_{2}, \ldots, p_{n-1}, p_{n}$ where $p_{n}$ is the predicate closest to the point of program failure, while $p_{1}$ is the predicate farthest from the point of failure. LEFS would therefore reorder $\sigma_{n}$ to $\sigma_{n}^{\prime}: p_{n}, p_{n-1}, \ldots, p_{2}, p_{1}$, with the result that the last encountered conditional branch is the first to be switched.

The detailed predicate switching procedure is described next. Given a WS-BPEL program BPEL, a failed test case $t$, its expected output $O$, and its associated LFES reordered predicate sequence $\sigma_{n}^{\prime}$ : $p_{n}, p_{n-1}, \ldots, p_{2}, p_{1}$, the following steps will be taken.

1. Set $i=n$, where $i$ is used to index the order of predicates in $\sigma_{n}^{\prime}$.

2. Mutate the WS-BPEL program BPEL by negating $p_{i}$ (e.g. change the Boolean value of $p_{i}$ from TRUE to FALSE, or vice versa) and derive the mutated WS-BPEL program BPELVariant.

3. Redeploy BPELVariant.

4. Execute BPELVariant with $t$ and obtain its output $O^{\prime}$

- If $O^{\prime}$ is the same as $O, p_{i}$ is identified as the critical predicate and the procedure is terminated;

- If $O^{\prime}$ is different from $O$ and $i$ is equal to 1 , there is no critical predicate and the procedure is terminated;

- Otherwise, decrease $i$ by one and go back to Step 2.

As an illustration, in Figure 4, the output remains incorrect until $p_{n-4}$ is switched. In other words, $p_{n-4}$ is the critical predicate. Note that it is possible that none 


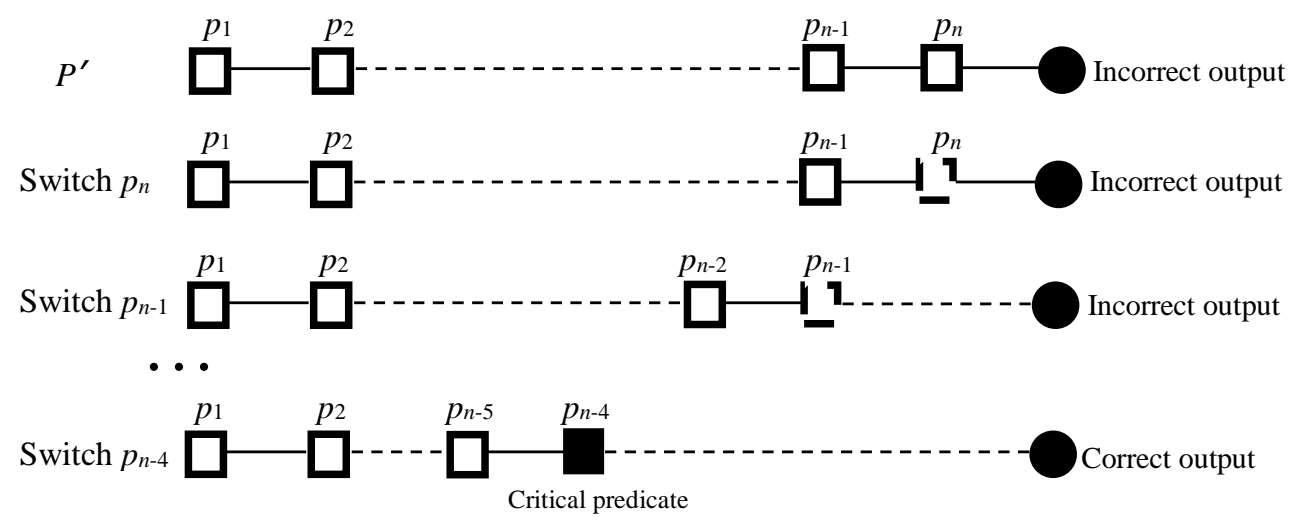

Figure 4: Illustration of searching critical predicate

of the predicates could be identified as the critical pred- ${ }_{481}$ icate even after all of them have been switched. In our ${ }_{482}$ BPELswice technique, the backward slicing will be im- ${ }_{483}$ plemented either from the critical predicate (if it is iden- ${ }^{484}$ tified) or from the end node of the program (if no critical 485 predicate is identified).

Unlike the original predicate switching, our method ${ }^{487}$ employs a mutation-based technique to implement pred- 488 icate switching, i.e. we first mutate a predicate in the 489 WS-BPEL program according to the LEFS strategy and 490 then redeploy the mutated WS-BPEL program. This 491 treatment is different from the implementation of pred- 492 icate switching for traditional programs. For instance, 493 a dynamic instrumentation technique was used to im- 494 plement predicate switching for C programs [11]. We 495 do not believe instrumentation is suitable for WS-BPEL programs, because it may introduce significant changes 496 to the original program and require modifications of the existing WS-BPEL engine, as discussed in Section 1.

\subsection{Execution}

During the execution process, we use an Apache ODE engine [13] to redeploy the WS-BPEL service. The ODE engine is capable of talking to Web services, sending and receiving messages, handling data manipulation and performing error recovery, as described in the process definition. It supports both long and short duration process executions to facilitate all services in an application, and enables WS-BPEL programs to be invoked as services. The execution process consists of 508 two steps: service deployment and process compiling. 509 During the service deployment, the package (including 510 all individual services and description files) is copied to 511 the server, where the ODE engine deploys the service ${ }_{512}$ and outputs the deploy.xml file. We used Apache Tomcat for this. The second step (process compiling) helps ensure that the service can be executed and invoked successfully. When a new WS-BPEL program variant is copied into the process directory of the engine, previous ones are deleted, and the engine redeploys it at once.

Communication between the client and server depends on Apache Axis2 [20], which encapsulates the test case in a soap message, and sends it to the server. After sending these messages, Axis2 parses and passes them to receiveInput in the WS-BPEL program. The engine then assigns values and invokes some services to complete the operation. As soon as the engine produces the output, Axis2 parses and encapsulates it into a soap message, and passes it to the client side.

\subsection{Evaluation}

Following the execution, an output is produced, and one of the following two situations exists: either this is the first execution, in which case the next step is to begin the switching process; or it is necessary to compare the output with what was expected to confirm whether or not the current predicate under evaluation is the critical predicate - output being consistent with expectation means that the critical predicate has been found. If the outputs remain different, then the critical predicate has not yet been found, and the predicate switching process continues.

\subsection{Slicing}

Once the critical predicate has been identified, we first examine whether the fault is located in the critical predicate. If yes, the fault localisation process can be terminated. Otherwise, the related program slices 
should be examined to localise the most suspicious statements related to the fault. The procedure for identifying these slices is discussed as follows.

The input of the slicing procedure includes

- $P(T)$, the execution trace when executing the program $P$ with a failed test case. $P(T)=<$ $X_{1}, X_{2}, \ldots, X_{i}, \ldots, X_{n}>$, where $X_{i}$ is a node in the execution path, $1 \leq i \leq n$, and $n$ is the total number of nodes in the path.

- $X_{q}$, the critical predicate, which is an element of $P(T)$, that is, $1 \leq q \leq n$.

- $U S\left(X_{i}\right)$, the set of def-use pairs of a node $X_{i}$. $\left.\left.U S\left(X_{i}\right)=\left\{<v_{d 1}^{i}, v_{u 1}^{i}\right\rangle,<v_{d 2}^{i}, v_{u 2}^{i}\right\rangle, \cdots\right\}$. It can be obtained from the parsing of WS-BPEL program and WSDL documents, as detailed in the following.

According to the basic concepts of data flow analysis [21], the def of a variable refers to the operation where a concrete value is allocated to the variable, while the use operation means that the variable is utilised either in a calculation ( $c$-use) or a predicate ( $p$-use). Since the data flow in WS-BPEL is different from that in traditional programming languages, we have the following definitions for the def and use in WS-BPEL programs.

- In WS-BPEL, the def of a variable normally happens at

- The Receive activity: the "variable" attribute,

- The Invoke activity: the "outputVariable" attribute, and

- The Assign activity: the left part of the expression in the "to" element.

556

- In WS-BPEL, the c-use of a variable normally hap- 558 pens at

- The Invoke activity: the "inputVariable" at- 560 tribute,

- The Reply activity: the "variable" attribute, and

- The Assign activity: the right part of the expression in the "from" element.

- In WS-BPEL, the p-use of a variable normally hap- ${ }^{567}$ pens at

- The Switch activity: the Boolean expression 570 in the "case" statement,

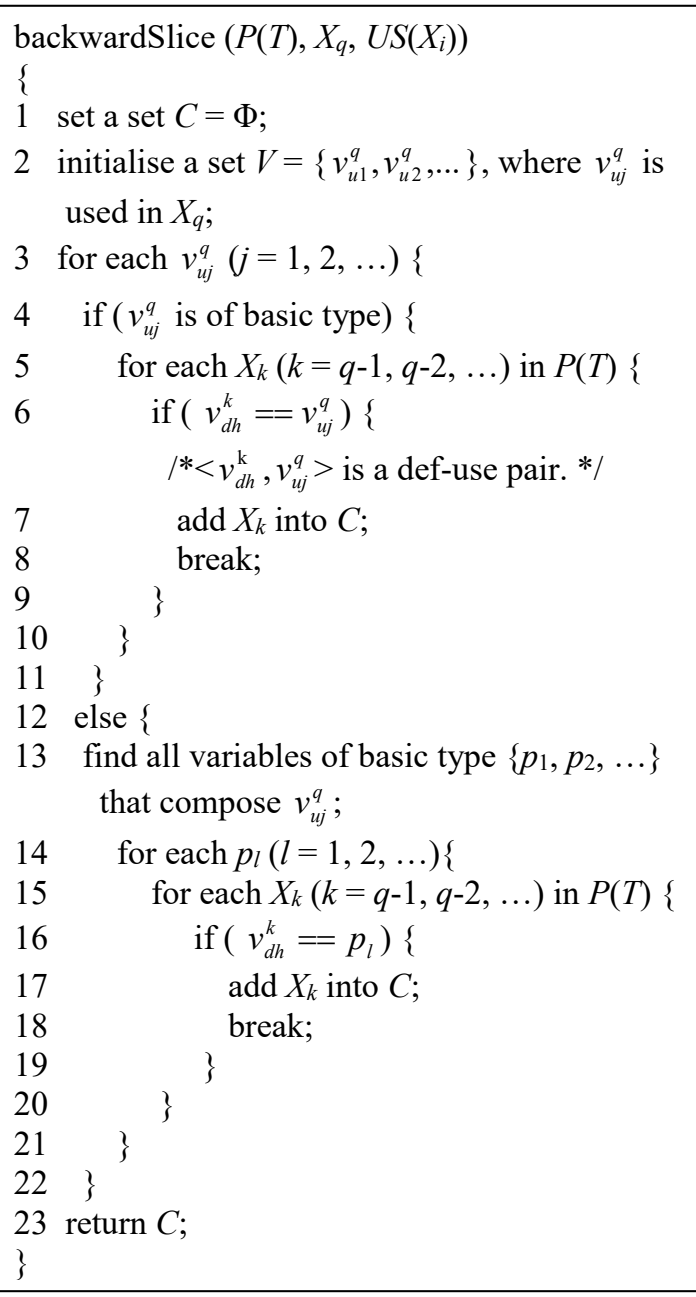

Figure 5: Procedure of backward slicing

- The While activity: the "condition" attribute, and

- The If activity: the "condition" attribute.

The basic procedure of backward slicing is as follows. Assume that the critical predicate is $X_{q}$, and all the variables used in $X_{q}$ are $\left\{v_{u 1}^{q}, v_{u 2}^{q}, \cdots\right\}$. For each $v_{u j}^{q}$, we search the nearest node backward (that is, first $X_{q-1}$, then $X_{q-2}, \ldots$ ) until we find the node $X_{k}$ where $v_{u j}^{q}$ (or the variables of basic type that comprise $v_{u j}^{q}$ ) is defined (that is, the latest definition of $v_{u j}^{q}$ before it is used in $X_{q}$ ). The collection of all $X_{k}$ will be the set of slices that are expected to contain the fault. The detailed backward slicing procedure is given in Figure 5. Note that, unlike programs written in traditional languages, WSBPEL programs normally involve variables of complex type. For a variable $v_{u j}^{q}$ of complex type, we need to first 
decompose it into variables of basic type $\left(\left\{p_{1}, p_{2}, \ldots\right\}\right.$ in 623 Figure 5), and find the nearest node where each $p_{l}$ is de- 624 fined. Such a process, as shown by Statements 12 to 21625 in Figure 5, is specific to WS-BPEL.

\subsection{Illustration}

We use the SmartShelf WS-BPEL program to illus- 629 trate how our BPELswice technique works. SmartShelf 630 is composed of 53 nodes and 13 services, as shown in 631 Figure 6. Every service uses some parameters which come from the WS-BPEL program ReceiveInput part: SmartShelf uses the three parameters named "name", "amount" and "status", the first two of which come from ReceiveInput, and the third from the ReadStatus.

For the CheckStatus service, SmartShelf invokes different services according to the variable "amount", and refers to the status to judge whether the product is expired or not, returning "Expired commodity has been replaced" or "Commodity is in good status", respectively. ${ }^{637}$ The CheckLocation service returns whether or not the product is available on the shelf. If not, it invokes another service to correct the location. The CheckQuantity service checks whether or not a sufficient amount of the good is available, returning either "Quantity is suffi- ${ }^{641}$ cient", or "Warehouse levels are insufficient, alert staff 642 to purchase", as appropriate.

The test case with "name"= candy, and "amount" ${ }_{644}$ $=100$, "candy\&\&100", can be passed as input to this 645 WS-BPEL service. Because of the initial settings in 646 the database, the executed flow structure sequence in- 647 volves the services "CheckStatus", "CheckLocation" 648 and "CheckQuantity". The executed runtime path is Path $_{1}=\left\{1-6,7-10,16-17,18-21,27-28,29-{ }^{649}\right.$ $32,33-37,38-42,48-53\}$, as obtained from the 650 ODE engine. Next, the predicate nodes are identi- 651 fied in the path: the predicate set in Path $_{1}$ is $\operatorname{Pre}_{1}={ }_{652}$ $\{10,21,32,37\}$. These predicates can then be switched. 653 In this study, we use the LEFS ordering strategy, which 654 reorders Pre $_{1}$ to Pre $_{1}^{\prime}=\{37,32,21,10\}$. The predicate 655 details for $P a t h_{1}$ are shown in Table 1.

When switching the predicates according to the order 657 in $\operatorname{Pre}_{1}^{\prime}$, two steps are involved. First, we identify the 658 target predicate, and switch its status (e.g., _status $=0659$ becomes_status $!=0$ ). Then, the previous WS-BPEL 660 file is deleted and replaced with this new one, and the ${ }_{661}$ ODE engine is used to redeploy the service.

After each predicate is switched, the same test case ("candy\&\&100") is input, and the output is recorded and compared with the expected output. Suppose that ${ }^{664}$ when the predicate with "location =0" (that is, If2 at 665 node 21) is switched to "location ! =0", the resulting 666 output becomes the same as the expected output - this 667 predicate is therefore the critical predicate, and is therefore suspected to be strongly connected to the fault. If the fault is found in this predicate, the localisation process can be successfully ended. Otherwise, some backward slices can be captured at runtime using the ODE engine. In this example, from the critical predicate (at node 21), we search backward and identify Assign16 (node 20), CheckLocation (node 18), and Assign (node 3 ) as the slices that are expected to contain the fault.

\section{Empirical Study}

We conducted a series of experiments to evaluate the performance of the proposed BPELswice technique. The empirical study was designed as follows.

\subsection{Research Questions}

Our empirical study was mainly focused on answering the following four research questions.

RQ1 How effectively does BPELswice localise the fault in a WS-BPEL program?

One critical criterion for evaluating the effectiveness of a fault localisation technique is whether or not it can successfully identify the statements/blocks that contain the fault. In our study, we applied BPELswice in the debugging of hundreds of mutants of three object programs, and then measured its effectiveness through examining how many faults BPELswice successfully localised.

RQ2 How precise is BPELswice in fault localisation?

In addition to the high fault localisation effectiveness, it is also important for a technique to have a high precision in the localisation. One naive way to maximise the high fault localisation effectiveness is to simply identify all executable statements in the program, which must contain the fault. Obviously, such a way is useless and very inefficient. In order to improve the efficiency of debugging, the number of statements identified by a fault localisation technique should be as small as possible, without eliminating the faulty statement. In our study, we measured the precision of BPELswice by evaluating how many statements it identified for each mutant.

RQ3 How quickly can BPELswice localise the fault?

If a technique is very time-consuming, its applicability would be greatly hindered. Therefore, it is necessary to investigate the execution time of 


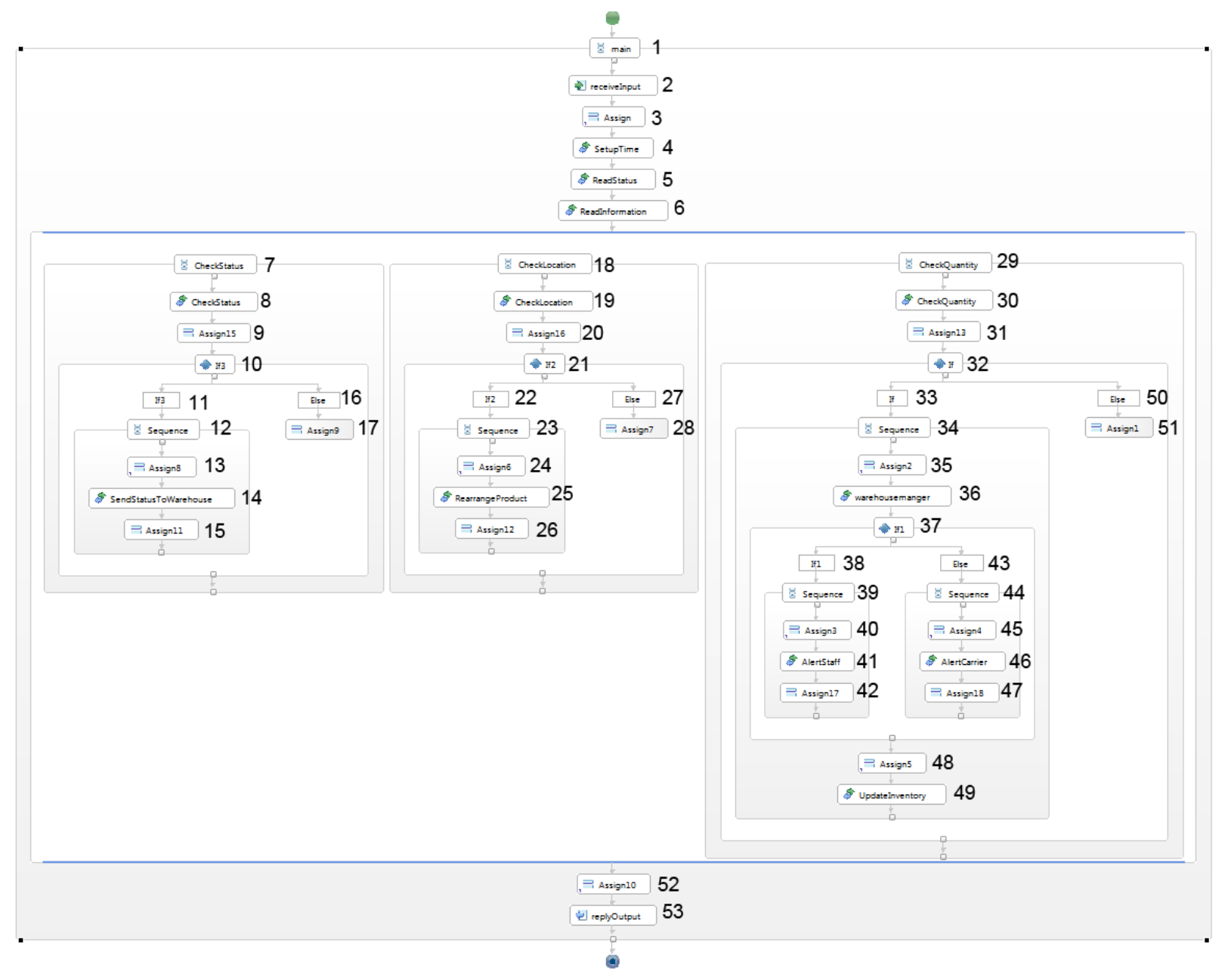

Figure 6: Structure of the SmartShelf WS-BPEL program

Table 1: SmartShelf's Predicate Set in $P_{a t h}$ for Test Case "candy\&\&100"

\begin{tabular}{|l|l|l|l|}
\hline If3 (node 10) & If2 (node 21) & If (node 32) & If1 (node 37) \\
\hline \$_status $=0$ & \$_location $=0$ & \$_amount $<$ \$init_amount & warehouseManagerReturn $<$ \$init_amount \\
\hline
\end{tabular}

the proposed BPELswice technique to see whether 679 it can provide a high effectiveness and precision within reasonable time.

RQ4 How many times does BPELswice need to switch ${ }^{682}$ predicates?

It can be naturally conjectured that the computational overhead in BPELswice is mainly related to the predicate switching process. In this study, ${ }_{687}$ we will evaluate the concrete number of predicate switches conducted by BPELswice on WS-BPEL programs.

\subsection{Variables and Measures}

\subsubsection{Independent variable}

The independent variable of our empirical study is the fault localisation technique. Our proposed BPELswice technique was selected for this variable. Since BPELswice is based on the predicate switching and backward slicing techniques, it is natural to select each of these two techniques (denoted switchOnly and sliceOnly in the rest of the paper) as the baseline techniques for comparison. In addition, we selected the Tarantula technique as another baseline technique for a better comparison with previous work [4]. As discussed in Section 2, 
Tarantula was the most effective fault localisation tech- 73 nique for WS-BPEL programs, compared with the Code Coverage and Set-Union techniques [4].

\subsubsection{Dependent variable}

The dependent variable relates to the measurement. In order to answer RQ1, we used the metric success rate to measure the fault localisation effectiveness. Given a number of faults, the success rate of a fault localisation technique is defined as the percentage of the number of successfully localised faults out of the total number of faults, that is,

$$
\text { success rate }=\frac{\text { number of localised faults }}{\text { total number of faults }} \times 100 \% \text {, }
$$

where a fault is said to be localised by a technique if the statement identified by the technique contains the fault. Obviously, the higher the success rate is, the more effective a technique is in fault localisation.

In order to answer RQ2, we measured the precision with the metric identification ratio. Given a fault, if a technique successfully localises it, the precision of the technique is defined as the percentage of the number of statements identified by the technique against the total number of statements of the program, that is,

$$
\begin{aligned}
& \text { identification ratio } \\
& \qquad=\frac{\text { number of identified statements }}{\text { total number of statements }} \times 100 \% \text {. }
\end{aligned}
$$

Intuitively speaking, the lower the identification ratio ${ }^{754}$ is, the more precise a technique is in fault localisation.

For RQ3, we made use of the runtime to measure how 756 fast a fault localisation technique can be. For the BPEL- 757 swice technique, the runtime is composed of the time 758 for finding the critical predicate and that for backward 759 slicing. Note that the execution time of the Tarantula 760 technique is not available, as the ranking of the pro- 761 gram elements is purely based on suspiciousness val- 762 ues, which are calculated based on the information at ${ }_{763}$ the testing stage. Therefore, we only compared the run- 764 time for BPELswice, switchOnly, and sliceOnly.

For RQ4, we measured the number of predicate 766 switches (denoted $N_{p s}$ ) that BPELswice requires when 767 it is used to localise a fault in a WS-BPEL pro- 768 gram. Note that there is no predicate switching process 769 in sliceOnly and Tarantula, and the switchOnly tech- 770 nique should have exactly the same $N_{p s}$ as BPELswice. 771 Hence, we will only present the $N_{p s}$ of BPELswice.

\subsection{Object Programs}

We selected three WS-BPEL programs, SmartShelf, TravelAgency, and QuoteProcess, as the objects in our empirical study. The basic information of these three programs is summarised in Table 2, which gives the lines of code (LOC), the number of implemented external services (\#Services), and the number nodes (\#Nodes) of each program. The SmartShelf program accepts the input parameters, including the name and amount of commodity, implements various services, and returns the status of commodity, the location of shelf, the quantity in warehouse, etc. The TravelAgency program is basically a booking system, involving the selection of travel plan, hotel reservation, ticket booking, and banking. The QuoteProcess program is used to simulate the user's selection of activities: it selects different activities according to user's input parameters. Note that TravelAgency is a sample WS-BPEL program, which was first introduced by OASIS [22], and is currently available at [23], while SmartShelf and QuoteProcess, on the other hand, were created by us according to third party business scenarios (available at [24]).

Table 2: Object programs

\begin{tabular}{|l|c|c|c|}
\hline Program & LOC & \#Services & \#Nodes \\
\hline SmartShelf & 579 & 13 & 53 \\
\hline TravelAgency & 427 & 6 & 24 \\
\hline QuoteProcess & 400 & 6 & 21 \\
\hline
\end{tabular}

\subsection{Mutant Generation}

For each object program, we generated a family of mutants using the MuBPEL tool [25]. Each mutant contains one and only one fault, which was seeded by applying a mutation operator into a certain statement. In MuBPEL, there are totally over 30 mutation operators [26]. However, not all the operators were applicable to each object program. As a matter of fact, due to the unique features of WS-BPEL, normally only a few mutation operators can be applied to a WS-BPEL program [27]. In our study, we used seven, nine, and seven mutation operators to generate mutants for SmartShelf, TravelAgency, and QuoteProcess, respectively. After the mutants were generated, we found that some could not be executed due to syntactic errors, so we eliminated them. There were also several so-called equivalent mutants, that is, they always showed the same execution behaviours as the basic programs. These equivalent mutants were also eliminated. Finally, our empirical study 
used 57, 56, and 53 mutants for SmartShelf, TravelA- 81 gency, and QuoteProcess, respectively. The basic mutant generation information is summarised in Table 3.

Table 3: Mutant generation

\begin{tabular}{|l|c|c|}
\hline Program & \#Operators & \#Mutants \\
\hline SmartShelf & 7 & 57 \\
\hline TravelAgency & 9 & 56 \\
\hline QuoteProcess & 7 & 53 \\
\hline
\end{tabular}

\subsection{Test Case Generation}

We used random testing to generate a large amount 826 of test cases. For a given WS-BPEL program, we first parsed the program to obtain the constraints on the input parameters. Random test data were generated for each input parameter, with the condition that the random ${ }^{828}$ data must satisfy the constraints. The random test case ${ }^{829}$ generation process was repeated until each fault was de- 830 tected by at least one test case. Here, a fault was consid- ${ }^{831}$ ered to be detected when a test case caused the relevant ${ }^{832}$ mutant to show different behaviour (more specifically, ${ }^{833}$ different output) to the basic program.

\subsection{Experiment Procedure}

Our empirical study was conducted on a laptop with a ${ }^{837}$ Windows 7 64-bit Operating System, an 8-core 3.4GHz 838 CPU (i7-4790), and 16G memory. The experiments 839 were run on Tomcat 6 and the ODE engine [13], which 840 can provide a lot of runtime information, including the ${ }^{841}$ execution path, predicates on the path, and the variable ${ }^{842}$ values. All this information can assist us in finding the ${ }^{843}$ fault. The basic procedure of the experiments is as fol- ${ }^{844}$ lows.

1. Start the ODE engine, and deploy one mutant of a WS-BPEL program.

2. Execute the test cases.

3. Obtain the critical information, including execution path, predicate sets, and actual output.

4. Find the test case that kills the mutant (that is, that causes the actual output to differ from the expected output).

5. Initiate predicate switching to identify the critical predicate.

6. If the critical predicate is identified, execute backward slicing from the critical predicate to localise the fault.

7. If the critical predicate is not identified, execute backward slicing from the wrong output.

8. Repeat the above steps until all mutants of each object program have been executed.

\subsection{Threats to Validity}

\subsubsection{Internal validity}

The main threat to internal validity relates to the implementation. The programming for implementing the BPELswice involved a moderate amount of work. Two of our authors conducted the programming work, one mainly for predicate switching, and the other mainly for slicing. All the source code was reviewed, crosschecked, and tested by different individuals. We are confident that the proposed BPELswice technique was correctly implemented, and thus the threat to internal validity has been minimised.

\subsubsection{External validity}

The threat to external validity is concerned with the selection of object programs and the fault types under study. In our study, we selected three representative WS-BPEL programs as the objects. These programs implement different functionalities, invoke different services, and have different scopes. Although we have endeavoured to maximise the diversity of object programs, we cannot guarantee that the results obtained from these three programs can be generally applied to any other WS-BPEL program. In addition, due to the nature of WS-BPEL programs, we could not study all possible fault types (mutation operators) for WS-BPEL, so it is also uncertain whether our conclusion is applicable to those fault types that were not investigated in this study. Moreover, it was assumed in this study that only one fault exists in a mutant. However, it would be very unlikely that the BPELswice technique could not work effectively for multiple faults - this is something that requires further empirical investigation.

\subsubsection{Construct validity}

There is little threat to construct validity in our study. The two metrics used in this study, success rate and identification ratio, are very straightforward in measuring the fault localisation effectiveness and precision.

\section{4.7.4. Conclusion validity}

In our experiments, we examined the performance of BPELswice based on 166 mutants of three object programs. A large amount of test cases were generated randomly. Therefore, a sufficient amount of experimental data was collected to guarantee the reliability of our results. In this sense, the threat to conclusion validity is very small. 


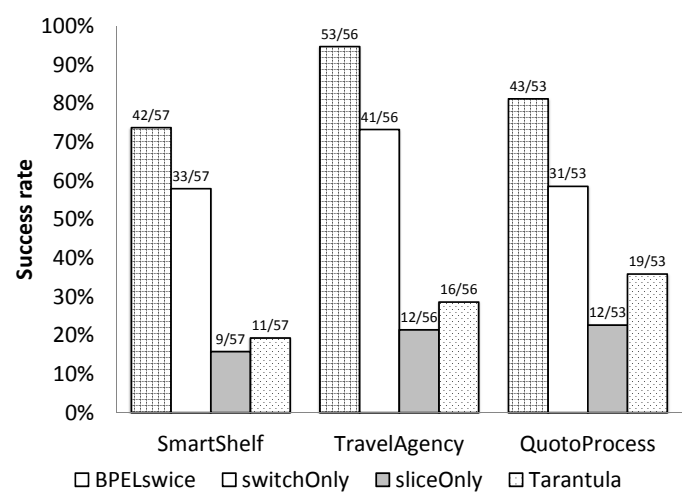

Figure 7: Comparison of success rates for BPELswice, switchOnly, sliceOnly, and Tarantula

\section{Results and analysis}

\subsection{RQ1: Effectiveness}

The experimental results of the success rates for BPELswice, switchOnly, sliceOnly, and Tarantula are given in Figure 7.

Based on Figure 7, we can observe that among 57, 56, and 53 faults (each in one mutant) for SmartShelf, TravelAgency, and QuoteProcess, respectively, BPELswice could successfully localise 42, 53, and 43 faults, giving success rates of $73.68 \%, 94.64 \%$, and $81.13 \%$, which were consistently the highest among the four fault localisation techniques. These results clearly show that BPELswice was much more effective than the other three techniques in the fault localisation for WS-BPEL programs.

We also investigated the faults that BPELswice failed to localise. We found that all these faults are of the types of "remove an activity" and "remove an element". Since BPELswice is based on the execution path, it cannot localise fault types related to "removal". For the same reason, Tarantula cannot localise these "removal" fault types either. In other words, all the faults localised by Tarantula were also successfully localised by BPELswice, but some faults localised by BPELswice could ${ }^{930}$ not be localised by Tarantula.

\subsection{RQ2: Precision}

The identification ratios for BPELswice, switchOnly, sliceOnly, and Tarantula are summarised in Figure 8. 936 In these figures, box plots are used to display the dis- ${ }_{937}$ tribution of identification ratios for one fault localisa- 938 tion technique on one object program. In each box, the 


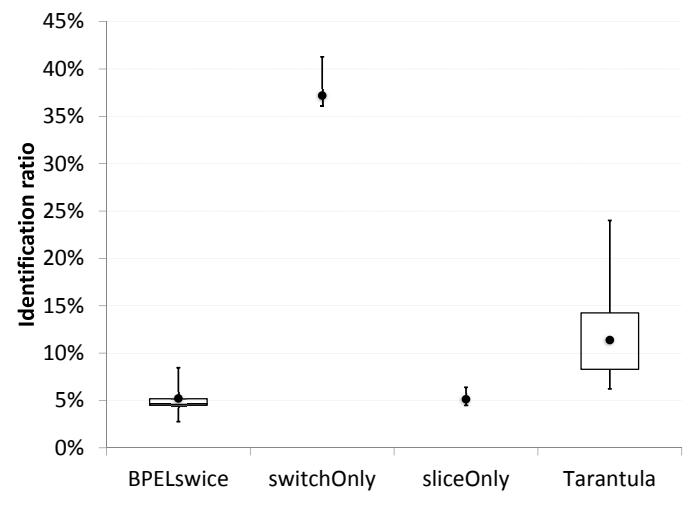

(a) SmartShelf

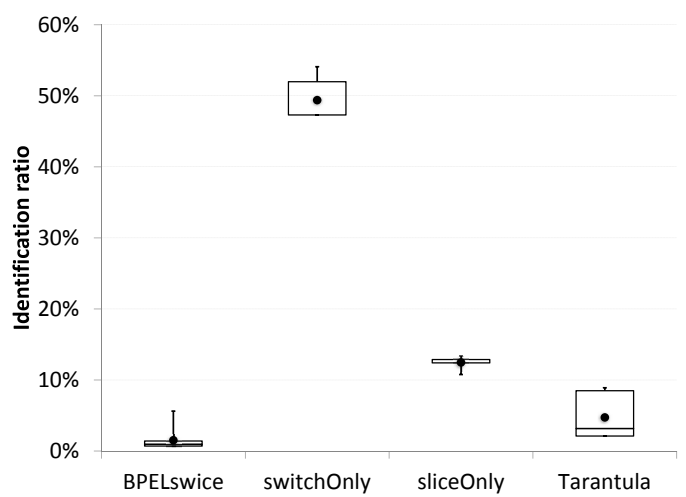

(b) TravelAgency

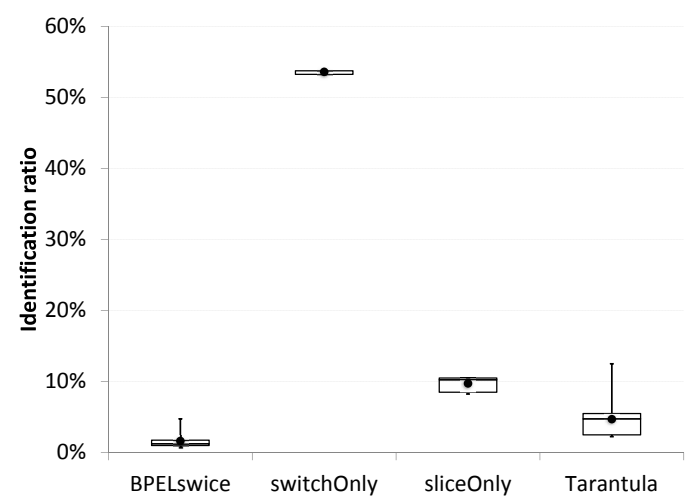

(c) QuoteProcess

Figure 8: Comparison of identification ratios for BPELswice, switchOnly, sliceOnly, and Tarantula

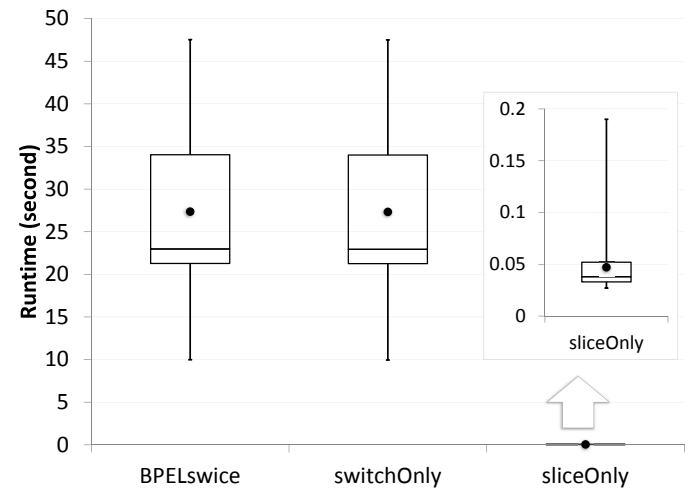

(a) SmartShelf

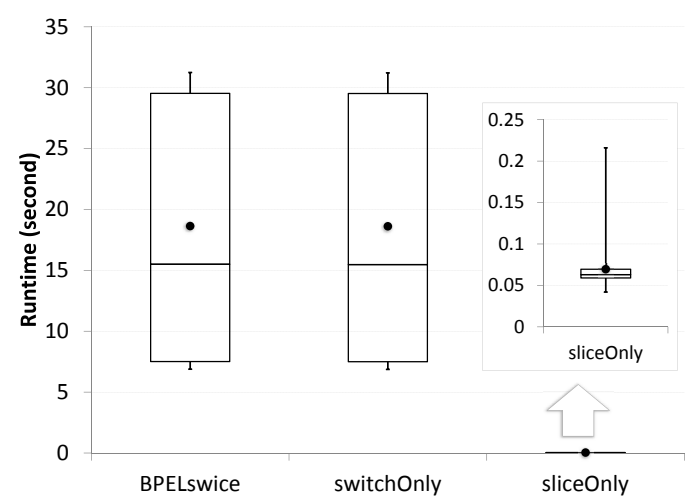

(b) TravelAgency

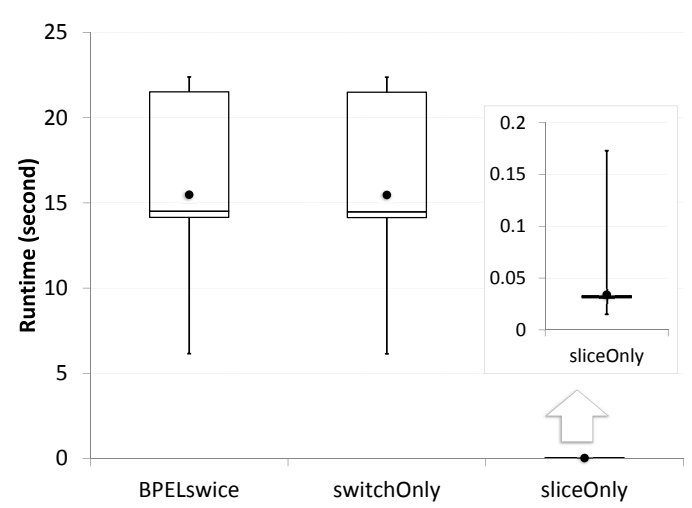

(c) QuoteProcess

Figure 9: Comparison of runtime for BPELswice, switchOnly, and sliceOnly 
executing BPELswice with the same $N_{p s}$. It can be ob- 972 served that BPELswice only needed to switch predi- 973 cates several times, with a maximum number of four 974 times. In addition, there is a strong correlation between 975 the value of $N_{p s}$ and the runtime, which is intuitively 976 as expected - the more predicates switched, the longer 977 BPELswice executed.

\section{Related work}

How to effectively locate faults reported by testing is ${ }^{982}$ a crucial activity in debugging. A lot of effort on this ${ }^{983}$ topic has been made and a number of fault localisation ${ }^{984}$ techniques have been proposed [16][28][17]. These ${ }^{985}$ techniques explore the fault localisation problem in dif- 986 ferent ways. The reported approaches include those ${ }^{987}$ based on program analysis, on program execution, and ${ }^{988}$ also using data mining or machine learning. Next, we ${ }^{989}$ discuss related work in terms of slicing-based fault lo- 990 calisation techniques, predicate-based fault localisation 991 techniques, and fault localisation techniques for WS- 992 BPEL programs.

\subsection{Slicing-based fault localisation techniques}

One category of fault localisation techniques is based on program analysis techniques such as program slicing [29], symbolic execution [30], and formal methods. Program slicing is the most widely used one for debugging aids. The principle of program slicing is to strip a program of statements without influence on a given variable at a given statement [29]. The idea of program slicing-based fault localisation is: given a program $p$ and a variable $v$ at a statement $i$ where a fault appears, the suspicious slice is the statements that directly affect the value of $v$ at $i-$ this eliminates those that have no

Table 4: $N_{p s}$ of BPELswice

\begin{tabular}{|c|c|c|c|}
\hline Program & $N_{p s}$ & \#Mutants & $\begin{array}{l}\text { Runtime range } \\
\text { (second) }\end{array}$ \\
\hline \multirow[t]{4}{*}{ SmartShelf } & 1 & 8 & {$[9.98,11.95]$} \\
\hline & 2 & 15 & {$[19.05,23.50]$} \\
\hline & 3 & 10 & {$[31.57,35.29]$} \\
\hline & 4 & 9 & {$[41.21,47.53]$} \\
\hline \multirow[t]{4}{*}{ TravelAgency } & 1 & 18 & {$[6.89,7.66]$} \\
\hline & 2 & 9 & {$[14.60,17.64]$} \\
\hline & 3 & 7 & {$[21.94,23.01]$} \\
\hline & 4 & 19 & {$[28.84,31.25]$} \\
\hline \multirow[t]{3}{*}{ QuoteProcess } & 1 & 9 & {$[6.16,8.27]$} \\
\hline & 2 & 19 & {$[13.36,15.13]$} \\
\hline & 3 & 15 & {$[21.41,22.39]$} \\
\hline
\end{tabular}

impact on the value of $v$ at $i$. A pioneering study was reported by Weiser [31] and showed the evidence that programmers slice when debugging.

Generally, program slicing can be either static or dynamic: the former is only based on the source code, while the latter works on a specific execution of the program (for a given execution trace). Fault localisation based on static program slicing analyzes the data flow and control flow of the program statically to reduce the search scope of faults [32], and its fault localisation precision is low since no other information than source code is used. Fault localisation based on dynamic program slicing introduces more precise slicing criteria for a particular execution and the search scope of faults can be further reduced [33]. Many efficient slicing algorithms have been proposed, and these algorithms may be used to further improve the efficiency of program slicing-based fault localisation techniques. For instance, $r o B D D$ is an efficient forward dynamic slicing algorithm using reduced ordered binary decision diagrams [34]. Recently, Wen [35] proposed program slicing spectrum to improve the effectiveness of statistical fault localisation methods, where the program slice is first used to extract dependencies between program elements and refine execution history, and then the suspiciousness of each slice is calculated to locate the fault based on statistical indices.

In our study, a backward dynamic program slicing technique was used to further improve the efficiency of locating faults in WS-BPEL programs. Our approach first analyzes the execution trace from an WS-BPEL engine and then extract suspicious statements via data flow analysis. Only those statements that have a direct impact on the value of elementary variables in the critical predicate are chosen. Our approach addressed the challenges due to the fact that the syntax, data structure, and execution mode of WS-BPEL programs are different from that of traditional programs.

\subsection{Predicate-based fault localisation techniques}

The other category of fault localisation techniques is based on program execution. Typically, such techniques make use of a program execution spectrum obtained in software testing to locate the suspicious elements. These techniques count the executions of program elements in different executions, and use the ratio of a program element being exercised in a failed execution and that in a passed execution to calculate the suspiciousness of the program element. Naish et al. [16] surveyed 33 different formulas for the suspiciousness calculation. The existing approaches work either at the level of statements or based on predicates. 
Fault localisation techniques at the level of state- 1075 ments, such as Tarantula [5] and Code Coverage [7], of- 1076 ten rely on statistics and need both successful and failing 1077 test cases to work. However, because they depend more 1078 on the pass or fail status of the test cases, and do not consider the static structure of the program, these methods may face other challenges. Renieris and Reiss [6] proposed a Set-Union technique based on neighboring queries which separated the failing program slices from 1081 the successful slice sets, deleting slices that appeared in 1082 both successful and failed execution paths, thereby gen- 1083 erating a suspicious statement set.

Fault localisation techniques based on predicates first 1085 instrument predicates in programs, and then capture 1086 and/or sample execution behaviours to efficiently iden- 1087 tify fault-relevant program elements. Among these 1088 techniques, some are based on statistics, and others 1089 are based on predicate switching. Typical predicate- 1090 based statistical fault localisation techniques include: 1091 Liblit et al. [36] ranked the predicates according to 1092 the probability that the program under study will fail 1093 when those predicates are observed to be true; Nainar 1094 et al. [37] used compound Boolean predicates to lo- 1095 cate faults; Zhang et al. [38] investigated the impact ${ }_{1096}$ of short-circuit evaluations on the effectiveness of ex- 1097 isting predicate-based techniques; Chilimbi et al. [39] 1098 used path profiles as fault predictors to locate faults; 1099 Hao et al. [40] proposed a self-adaptive fault localisa- ${ }_{1100}$ tion algorithm which dynamically selects the intensity 1101 of each predicate based on predicate execution informa- 1102 tion analysis.

Predicate-switching based fault localisation was first proposed by Zhang et al. [11]: it focuses on a failed run corresponding to single input for fault localisation. Un- 1104 like existing statistical techniques, the idea of this technique is to forcibly switch a predicate's outcome at run- 1105 time and alter the control flow until the program pro- 1106 duces the desired output. By examining the switched ${ }_{1107}$ predicate, the cause of the fault can then be identi- 1108 fied. Although predicate-switching based fault local- 1109 isation significantly reduces the search space of po- 1110 tential state changes, the overhead for locating a pro- 1111 gram with scaled predicates may still be high. Wang 1112 and Liu [41] proposed a hierarchical multiple predicate 1113 switching method which restricts the search for criti- 1114 cal predicates to the scope of highly suspect functions 1115 identified by employing spectrum-based fault localisa- 1116 tion techniques. The predicate switching technique has 1117 demonstrated good efficiency for locating faults in $C_{1118}$ programs.

In our study, the predicate switching technique was 1120 employed to narrow the search scope of blocks within 1121
WS-BPEL programs. In particular, we implemented the predicate switching technique through mutating predicates rather than instrumentation, which is very different from previous studies $[11,41]$.

\subsection{Fault localisation techniques for WS-BPEL pro- grams}

As mentioned before, WS-BPEL programs demonstrate new features that are not common in traditional programs, and accordingly suffer from new fault types. In our previous work [4], we explored the fault localisation issue of WS-BPEL programs and proposed a block-based fault localisation framework. We synthesized three well-known spectrum-based fault localisation techniques within the framework (Tarantula [5], Set-Union [6], and Code Coverage [7]), and evaluated the effectiveness of the synthesized techniques using two WS-BPEL programs. Although such techniques were empirically evaluated to be effective in previous studies [18], however, their effectiveness was not as good as expected when they were used for the fault localisation of WS-BPEL programs.

In this study, we addressed the above problem with a new fault localisation technique for WS-BPEL programs which combines predicate switching with program slicing. We empirically evaluated and compared the effectiveness and precision of the proposed technique with the Tarantula technique, which showed the best performance in the synthesized techniques for WSBPEL programs in our previous work.

\section{Conclusion}

WS-BPEL program have many new features and also suffer from new types of faults when compared with traditional programs that are written in $\mathrm{C}, \mathrm{C}++$, or Java. In this paper, we have presented a novel fault localisation technique, BPELswice, for WS-BPEL programs. The proposed technique is composed of two main components: the predicate switching method, which is used to greatly reduce the state search space of faulty codes through looking for so-called critical predicates, and the dynamic backward slicing method, which is used to improve the fault localisation precision through dataflow analysis of execution traces of WS-BPEL programs. Three case studies were conducted to evaluate the fault localisation performance of the proposed technique in terms of correctness and precision, and compare its performance with that of predicate switching only, slicing only, and Tarantula, which was considered to be the 
most effective one for WS-BPEL programs. The experi- 1171 mental results show that the proposed BPELswice tech- 1172 nique had a higher fault localisation effectiveness and precision than predicate switching only, slicing only, and Tarantula. In other words, this study proposes 1173 a more effective fault localisation technique for WSBPEL programs.

This study advances the state of the art for the fault ${ }^{1175}$ localisation of WS-BPEL programs in the following ${ }_{1177}^{1176}$ ways: (i) we propose a new fault localisation framework 1178 to further improve the fault localisation effectiveness of ${ }^{1179}$ WS-BPEL programs, considering new features of WS- ${ }^{1180}$ BPEL programs (i.e. a new style of programs); (ii) we ${ }_{1182}^{1181}$ address the challenging issues related to when predicate 1183 switching is used for WS-BPEL programs, where the ${ }^{1184}$ predicate switching mechanism is very different from ${ }_{1186}{ }_{1185}$ that which was developed for C programs [11]; (iii) we ${ }_{1187}^{1186}$ report on the technical treatment of the backward dy- 1188 namic slicing technique for WS-BPEL programs, which ${ }^{1189}$ is significantly different from that for traditional pro- ${ }_{1190}^{1190}$ grams; (IV) we provide a comprehensive evaluation ${ }_{1192}$ and comparison of the proposed technique with exist- ${ }^{1193}$ ing techniques in this field.

In our future work, we are interested in the follow- ${ }_{1196}$ ing directions: (i) extending the proposed framework to ${ }_{1197}$ cover other sections of WS-BPEL programs (the current ${ }^{1198}$ one only consider the faults in the interaction section ${ }_{1200}^{1199}$ of WS-BPEL programs); (ii) developing techniques to ${ }_{1201}^{200}$ enable isolation of the faults in the level of WS-BPEL 1202 programs or invoked services; and (iii) investigating the ${ }^{1203}$ differentiation of types of locating fault among the dif- ${ }^{1204}$ ferent fault localisation techniques.

\section{Acknowledgements}

This research is supported by the National Nat- ${ }^{1212}$ ural Science Foundation of China under Grant No. ${ }^{1213}$ 61370061, the Beijing Natural Science Founda- ${ }^{1214}$ tion (Grant No. 4162040), the Beijing Municipal ${ }_{1216} 215$ Training Program for Excellent Talents under Grant 1217 No.2012D009006000002, and the Aeronautical Science ${ }^{1218}$ Foundation of China (Grant No. 2016ZD74004). Dave ${ }_{1220}^{1219}$ Towey acknowledges the financial support from the Ar- ${ }_{1221}$ tificial Intelligence and Optimisation Research Group of 1222 the University of Nottingham Ningbo China, the Inter- ${ }^{1223}$ national Doctoral Innovation Centre, the Ningbo Edu- ${ }_{1225} 224$ cation Bureau, the Ningbo Science and Technology Bu- ${ }_{1226}$ reau, and the University of Nottingham. The authors are ${ }^{1227}$ grateful to $\mathrm{An} \mathrm{Fu}$ and Cuiyang Fan from University of ${ }^{1228}$ Science and Technology Beijing for their help in con- ${ }_{1230}{ }^{2229}$ ducting extra experiments reported in this work, and to ${ }_{1231}$ the anonymous referees for their helpful comments on an earlier version of this paper.

\section{References}

[1] M. Papazoglou, P. Traverso, S. Dustdar, F. Leymann, Serviceoriented computing: A research roadmap, International Journal on Cooperative Information Systems 17 (2) (2008) 223-255.

[2] Eviware, Web Services Business Process Execution Language Version 2.0, http://docs.oasis-open.org/wsbpel/2.0/ OS/wsbpel-v2.0-0S.html (2012).

[3] W3C, Extensible Markup Language (XML), http://www . w3. org/XML/ (2008).

[4] C.-A. Sun, Y. M. Zhai, Y. Shang, Z. Zhang, BPELDebugger: An effective BPEL-specific fault localization framework, Information and Software Technology 55 (12) (2013) 2140-2153.

[5] J. A. Jones, Fault localization using visualization of test information, in: Proceedings of 26th International Conference on Software Engineering, IEEE Computer Society, 2004, pp. 5456

[6] M. Renieres, S. P. Reiss, Fault localization with nearest neighbor queries, in: Proceedings of 18th IEEE International Conference onAutomated Software Engineering, IEEE, 2003, pp. 30-39.

[7] W. E. Wong, Y. Qi, L. Zhao, K.-Y. Cai, Effective fault localization using code coverage, in: Proceedings of 31st Annual InternationalComputer Software and Applications Conference (COMPSAC 2007), Vol. 1, IEEE, 2007, pp. 449-456.

[8] L. Zhao, Z. Zhang, L. Wang, X. Yin, Pafl: Fault localization via noise reduction on coverage vector, in: Proceedings of the Twenty-Third International Conference on Software Engineering and Knowledge Engineering(SEKE 2011), 2011, pp. 203206.

[9] Rose India, Activebpel designer v1.0, http://www.roseindia.net/eclipse/plugins/webservices/ActiveBPELDesigner.shtml.

[10] Eclipse, Bpel designer v1.0, http://www.eclipse.org/bpel/.

[11] X. Zhang, N. Gupta, R. Gupta, Locating faults through automated predicate switching, in: Proceedings of 28th international conference on Software engineering, ACM, 2006, pp. 272-281.

[12] B. Xu, J. Qian, X. Zhang, Z. Wu, L. Chen, A brief survey of program slicing, ACM SIGSOFT Software Engineering Notes 30 (2) (2005) 1-36.

[13] Apache, Apache ODE, http://ode.apache.org/ (2006).

[14] H. Haas and A. Brown, W3C, Web Services Glossary, http: //www.w3.org/TR/ws-gloss/ (2004).

[15] M. N. Huhns, M. P. Singh, Service-oriented computing: Key concepts and principles, IEEE Internet Computing 9 (1) (2005) 75-81.

[16] L. Naish, H. Lee, K. Ramamohanarao, A model for spectrabased software diagnosis, ACM Transactions on Software Engineering and Methodology 20 (3) (2011) 11:1-11:32.

[17] W. Wong, R. Gao, Y. Li, R. Abreu, F. Wotawa, A survey on software fault localization, IEEE Transactions on Software Engineering (2016) in press.

[18] J. A. Jones, M. J. Harrold, Empirical evaluation of the tarantula automatic fault-localization technique, in: Proceedings of the 20th IEEE/ACM International Conference on Automated Software Engineering (ASE 2005), 2005, pp. 273-282.

[19] Java Platform, Standard Edition 7 API Specification, http://docs.oracle.com/javase/7/docs/api/javax/ swing/JTree.html (2016).

[20] Apache, Apache Axis2, http://axis.apache.org/axis2/ java/core/ (2012). 
[21] U. Khedker, A. Sanyal, B. Sathe, in: Data Flow Analysis: Theory and Practice, CRC Press, 2009.

[22] OASIS, Web Services Business Process Execution Language Version 2.0, http://docs .oasis-open.org/wsbpel/2.0/ OS/wsbpel-v2.0-0S.html (2007).

[23] Linagora, Travel Agency, https://research.linagora. com/display/easiestdemo/Travel+Agency (2016).

[24] ActiveVOS, ActiveVOS sample applications, http://www. activevos.com/developers/sample-apps (2017).

[25] A. Estero-Botaro, F. Palomo-Lozano, I. Medina-Bulo, J. Dominguez-Jimenez, A. Garcia-Dominguez, Quality metrics for mutation testing with applications to WS-BPEL compositions, Software Testing, Verification and Reliability 25 (5-7) (2014) 536-571.

[26] A. Estero-Botaro, F. Palomo-Lozano, I. Medina-Bulo, Mutation operators for WS-BPEL 2.0, in: Proceedings of 21th International Conference on Software \& Systems Engineering and their Applications, 2008.

[27] C.-A. Sun, L. Pan, Q. Wang, H. Liu, X. Zhang, An empirical study on mutation testing of WS-BPEL programs, The Computer Journal 60 (1) (2017) 143-158.

[28] X. Xie, T. Y. Chen, F.-C. Kuo, B. Xua, A theoretical analysis of the risk evaluation formulas for spectrum-based fault localization, ACM Transactions on Software Engineering and Methodology 22 (4) (2013) 31:1-31:40.

[29] M. Weiser, Program slicing, IEEE Transactions on Software Engineering 10 (4) (1984) 352-357.

[30] J. C. King, A symbolic execution and program testing, Communications of the ACM 19 (7) (1976) 385-394.

[31] M. Weiser, Programmers use slices when debugging, Communications of the ACM 25 (7) (1982) 446-452.

[32] J. R. Lyle, M.Weiser, Automatic program bug location by program slicing, in: Proceedings of the Second International Conference on Computers and Applications, 1987, pp. 877-883.

[33] H. Agrawal, R. A. DeMillo, E. H. Spafford, Debugging with dynamic slicing and backtracking, Software - Practice and Experience 23 (6) (1993) 589-616.

[34] X. Zhang, R. Gupta, Y. Zhang, Efficient forward computation of dynamic slices using reduced ordered binary decision diagrams, in: Proceedings of 26th International Conference on Software Engineering, IEEE Computer Society, 2004, pp. 502-511.

[35] W. Wen, Software fault localization based on program slicing spectrum, in: Proceedings of 34th International Conference on Software Engineering, IEEE Press, 2012, pp. 1511-1514.

[36] B. Liblit, M. Naik, A. Zheng, A. Aiken, M. Jordan, Scalable statistical bug isolation, in: Proceedings of the 2005 ACM SIGPLAN Conference on Programming Language Design and Implementation (PLDI 2005), 2005, pp. 15-26.

[37] P. Nainar, T. Chen, J. Rosin, B. Liblit, Statistical debugging using compound boolean predicates, in: Proceedings of the 2007 ACM SIGSOFT International Symposium on Software Testing and Analysis (ISSTA 2007), 2007, pp. 5-15.

[38] Z. Zhang, B. Jiang, W. Chan, T. Tse, X. Wang, Fault localization through evaluation sequences, Journal of Systems and Software 83 (2) (2010) 174-187.

[39] T. Chilimbi, B. Liblit, K. Mehra, A. Nori, K. Vaswani, Holmes: effective statistical debugging via efficient path profiling, in: Proceedings of the 31st International Conference on Software Engineering (ICSE 2009), 2009, pp. 34-44.

[40] P. Hao, Z. Zheng, Z. Zhang, Self-adaptive fault localization algorithm based on predicate execution information analysis, Chinese Journal of Computers (2014) 500-510.

[41] X. Wang, Y. Liu, Automated fault localization via hierarchical multiple predicate switching, Journal of Systems and Software 104 (2015) 69-81. 